\title{
ISOPERIMETRIC-TYPE INEQUALITIES FOR GENERALIZED CENTROID BODIES
}

\author{
KeJIE ShI AND GANGSONG LENG
}

Abstract. We extend the Orlicz centroid body for probability measures to multivariate case and establish the affine isoperimetric-type inequality for the generalized centroid body. Moreover, the concepts of centroid body and mean zonoid are unified through the generalized definition, which is much more significant.

Mathematics subject classification (2010): 52A20, 52A40, 60D05.

Keywords and phrases: Generalized centroid body, probability measures, Orlicz centroid body, isoperimetric inequality, mean zonoid.

\section{REFERENCES}

[1] Z. Artstein, R. A. Vitale, A strong law of large numbers for random compact sets, Ann. Probab. 3 (1975), 879-882.

[2] S. CAmpi, P. Gronchi, The L L -Busemann-Petty centroid inequality, Adv. Math. 167 (2002), 128 141.

[3] R. M. Dudley, Real Analysis and Probability, Cambridge: Cambridge University Press, 2002.

[4] R. J. Gardner, Geometric Tomography, Cambridge: Cambridge University Press, 2006.

[5] R. J. Gardner, D. Hug, W. Weil, The Orlicz-Brunn-Minkowski theory: A general framework, additions, and inequalities, J Differential Geom. 94 (2014), 427-476.

[6] A. Giannopoulos, P. Stavrakakis, A. Tsolomitis, B. VRitsiou, Geometry of the $L_{q}$ centroid bodies of an isotropic log-concave measure, Trans. Amer. Math. Soc. 367 (2015), 4569-4593.

[7] L. Guo, G. Leng, C. Du, The Orlicz mean zonoid operator, J. Math. Anal. Appl., 424 (2015), 1261-1271.

[8] C. Haberl, E. Lutwak, D. Yang, G. Zhang, The even Orlicz Minkowski problem, Adv. Math. 224 (2010), 2485-2510.

[9] Q. HuAng, B. He, An asymmetric Orlicz centroid inequality for probability measures, Sci. China Math. 57 (2014), 1193-1202.

[10] B. Klartag, E. Milman, Centroid bodies and the logarithmic Laplace transform-Aunified approach, J. Funct. Anal. 262 (2012), 10-34.

[11] A. Li, G. Leng, A new proof of the Orlicz Busemann-Petty centroid inequality, Proc. Amer. Math. Soc. 139 (2011), 1473-1481.

[12] E. LutwaK, On some affine isoperimetric inequalities, J. Differential Geom. 23 (1986), 1-13.

[13] E. Lutwak, Centroid bodies and dual mixed volumes, Proc. London Math. Soc. 60 (1990), 365-391.

[14] E. LutwaK, D. YANG, G. ZHANG, $L_{p}$ affine isoperimetric inequalities, J Differential Geom. 56 (2000), 111-132.

[15] E. LutwaK, D. Yang, G. Zhang, A new ellipsoid associated with convex bodies, Duke Math. J. 104 (2000), 375-390.

[16] E. LutwaK, D. Yang, G. Zhang, Orlicz centroid bodies, J. Differential Geom. 84 (2010), 365-387.

[17] E. LutwaK, D. Yang, G. Zhang, Orlicz projection bodies, Adv. Math. 223 (2010), 220-242.

[18] E. Lutwak, G. Zhang, Blaschke-Santaló inequalities, J. Differential Geom. 47 (1997), 1-16.

[19] H. Martini, Z. Mustafaev, On isoperimetric inequalities in Minkowski spaces, J. Inequal. Appl. 2010, Art. ID 697954, 18 pp. 
[20] I. Molchanov, Convex and star-shaped sets associated with multivariate stable distributions. I. Moments and densities, J. Multivariate Anal. 100 (2009), 2195-2213.

[21] G. PAOuris, Concentration of mass on convex bodies, Geom. Funct. Anal. 16 (2006), 1021-1049.

[22] G. PAOURIS, On the existence of supergaussian directions on convex bodies, Mathematika, 58 (2012), 389-408.

[23] G. Paouris, P. Pivovarov, A probabilistic take on isoperimetric-type inequalities, Adv. Math. 230 (2012), 1402-1422.

[24] G. PaOuris, P. Pivovarov, Small-ball probabilities for the volume of random convex sets, Discrete Comput. Geom. 49 (2013), 601-646.

[25] C. M. PetTy, Centroid surfaces, Pacific J. Math. 11 (1961), 1535-1547.

[26] R. SchneIder, Convex Bodies: The Brunn-Minkowski Theory, 2nd ed. Cambridge: Cambridge University Press, 2014.

[27] D. XI, L. Guo, G. LenG, Affine inequalities for $L_{p}$ mean zonoids, Bull. Lond. Math. Soc. 46 (2014), 367-378.

[28] D. XI, H. Jin, G. Leng, The Orlicz Brunn-Minkowski inequality, Adv. Math. 260 (2014), 350-374.

[29] G. ZHANG, Restricted chord projection and affine inequalities, Geom. Dedicata 39 (1991) 213-222.

[30] G. Zhang, Centered bodies and dual mixed volumes, Trans. Amer. Math. Soc. 345 (1994), 777-801.

[31] G. ZHu, The Orlicz centroid inequality for star bodies, Adv. in Appl, Math. 48 (2012), 432 - 445. 